\title{
Strongly Asymmetric Doping Profiles at Mask Edges in High-Energy Ion Implantation
}

\author{
RUTGER C. WIJBURG, GERTJAN J. HEMINK, AND JAN MIDDELHOEK
}

\begin{abstract}
The application of high energy ion implantation is restricted by an asymmetric doping profile at the mask edges. As a result, buried interconnect cannot easily be formed. Moreover, the holding voltage and threshold voltage of CMOS-processes with retrograde wells may be strongly affected by this asymmetry. It arises from the $7^{\circ}$ wafer tilt, which is frequently used to avoid channeling, even in the case of nearly perpendicular $\left(82-85^{\circ}\right)$ mask edges. On the mask side, which is incoming to the ion beam, a trunk to the surface has experimentally been observed. According to 2-D-Gaussian and advanced Monte Carlo simulations, the doping concentration in this trunk is about 20 percent of the maximum concentration in the case of a $85^{\circ}$ mask angle. The simulations are fairly well able to predict the experimental results. The asymmetry effect of high energy ion implantations can also be visualized in photoresist by means of a damaged region.
\end{abstract}

\section{INTRODUCTION}

$\mathrm{H}$ IGH ENERGY ion implantations are frequently used in CMOS technology for VLSI applications. It offers the advantage, that a retrograde $n$-well or p-well can be formed after the high temperature step for field-oxidation [1]-[4]. In this way, the lateral diffusion of the created tub is strongly suppressed, thus reducing the necessary well layout. A retrograde well has the additional advantage of a low latch-up susceptibility. Both the low sheet resistance in the tub and the retrograde doping profile reduce the performance of the parasitic vertical bipolar transistor [4]. High energy implantations can also be used to replace conventional buried layers in small vertical RAM or ROM cells [5]-[7]. For these applications high energy implanted buried interconnect in active areas may offer good opportunities.

In this paper, it will be shown, that in the case of high energy ion implantation a strongly asymmetric doping profile at the mask edges arises in consequence of the 7-deg wafer tilt. This wafer tilt in the implanter is used to avoid ion channeling. Although, the critical angle for axial and planar channeling has a complicated dependence on parameters like type of ion, implantation energy and crystal orientation [8], [9], industrial (high energy) implantations frequently use a 7 -deg tilt to avoid channeling [10]-[12]. In fact the channeling problem can only be suppressed by implanting in a preamorphized layer [11]. That implies, that the high energy ion implantation is not

Manuscript received December 10, 1988; revised April 4, 1989. The review of this paper was arranged by Associate Editor B. Ricco.

The authors are with the Department of Electrical Engineering, University of Twente, $7500 \mathrm{AE}$ Enschede, The Netherlands.

IEEE Log Number 8930316. simply applicable for formation of buried interconnect in active areas, even if perpendicular mask edges are used. However, the wafer tilt also has its influence on the high energy ion implanted retrograde wells under the field-oxide. Recently, it has been reported, that the shadowing of thick mask layers result in a displacement in the position of the well [13]. Moreover, we found that the $7^{\circ}$ wafer tilt causes a strong asymmetric doping profile. This has a large influence on the threshold voltage of the field oxide and the holding voltage of the parasitic thyristor structures, especially when the maximum impurity concentration is not situated just under the field oxide.

In both experiments and simulations we show the detrimental influence of the wafer tilt on the high energy implanted doping profile. For the experiments, wafers with thick mask structures are implanted with $1 \mathrm{MeV}$ phosphorus ions. Both photoresist and anisotropical etched oxide serve as a mask for implantation. The experimental setup will be described in more detail in the next section. Simulations are performed to examine the dependence of the impurity profile on the mask angle and the wafer tilt angle. The doping concentration is calculated by a 2-DGaussian distribution and by an advanced Monte Carlo simulation. This Monte Carlo simulation also takes into account the ions which scatter from the mask into vacuum. Two programs were developed: IMPCAL which uses the 2-D-Gaussian approximation to compute the doping profile and SIMON which is a 2-D-Monte Carlo simulation program optimized to compute doping profiles near rectilinear mask edges. Both programs use silicon as masking material.

\section{Sample Preparation}

In the experiments, described here, both photoresist (type- $\mathrm{R}$ ) and oxide (type- $\mathrm{O}$ ) serve as a mask against implantation. Because of the high energy used, the mask should be rather thick to prevent ions from penetrating into the underlaying silicon. Three-inch wafers, boron doped to a concentration of $6 \times 10^{16} \mathrm{~cm}^{-3}$, are used.

On the type-R wafers a thermal oxide of a thickness of $80 \mathrm{~nm}$ is grown and the wafers are coated with a thick S1400-37 photoresist layer. The thickness of the photoresist layer, as measured by a Sloan Dektak 3030, is 3.3 $\mu \mathrm{m}$. Then, the wafers get a soft bake for $5 \mathrm{~min}$ on a hot plate to remove almost all the solvents from the photoresist. The soft bake temperature is $90^{\circ} \mathrm{C}$. After exposure in 


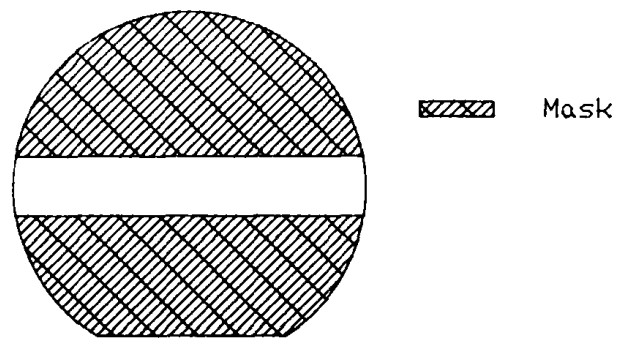

Fig. 1. The mask structure at a 3-in wafer made in order to create a incoming and shadow side to the ion beam.

a Canon wafer stepper FPA-1550 and development of the resist, no post baking is applied to preserve the maximum steepness of the photoresist mask edges. Two long photoresist mask edges are created as visualized in Fig. 1. For inspection of the photoresist in a SEM, also some wafers with many long photoresist lines are made, using exactly the same procedure.

The type- $\mathrm{O}$ wafers are oxidized at $1050^{\circ} \mathrm{C}$ in a wet oxygen ambient for $10 \mathrm{~h}$. This results in $1-\mu \mathrm{m}$ thick oxide. On top of it, another $1-\mu \mathrm{m}$ thick phosphorus and boron rich oxide layer is deposited in a CVD-reactor. Directly after deposition, the wafers are annealed at $900^{\circ} \mathrm{C}$ for 30 min in a nitrogen ambient. The total oxide thickness, measured with an ordinary ellipsometer, is $2.1 \mu \mathrm{m}$. A thin photoresist structure is made (comparable to the one shown in Fig. 1) and the wafers are etched in a RIE-reactor with a $\mathrm{CHF}_{3}$-plasma. The power density is 0.55 $\mathrm{W} \cdot \mathrm{cm}^{-2}$ and the $\mathrm{CHF}_{3}$-flow $10 \mathrm{sccm}$. In order to obtain fully anisotropical edges no oxygen is added during etching. The pressure is kept at a low value of $13 \times 10^{-6}$ bar to avoid polymerization. The etching process is controlled by an interferometer. Finally the thin photoresist layer is removed.

Both type-P and type-O wafers are placed in a high energy implanter with a $7^{\circ}$ wafer tilt (see Fig. 2). In this way, the mask edge at the left side lays more or less in the shadow of the incoming beam, whereas the mask edge on the right side is more receptive to the incoming beam. Further, these edges will be denoted as shadow side and incoming side, respectively. The sweep of the ion beam scanning over the 3-in wafers may introduce a deviation from the $7^{\circ}$ tilt. However, the mask edges are closely situated to the middle of the wafer and thus rather centered in the ion beam sweep. Therefore, the deviation from the $7^{\circ}$ tilt will be less than $0.4^{\circ}$.

Phosphorus ions with an energy of $1 \mathrm{MeV}$ are implanted up to a maximum dose of $2 \times 10^{13} \mathrm{~cm}^{-2}$. After removing the photoresist layer from the type- $\mathrm{R}$ wafers, an anneal step of $30 \mathrm{~min}$ at $800^{\circ} \mathrm{C}$ in a nitrogen ambient is carried out in order to remove the implantation damage and to activate the implanted phosphorus ions. The wafers are etched in BHF to remove any oxide. Thereafter, the silicon surface is ball-grooved at several places along the lines, where the shadow and incoming mask edges were situated. These grooves are stained in an etch, which is

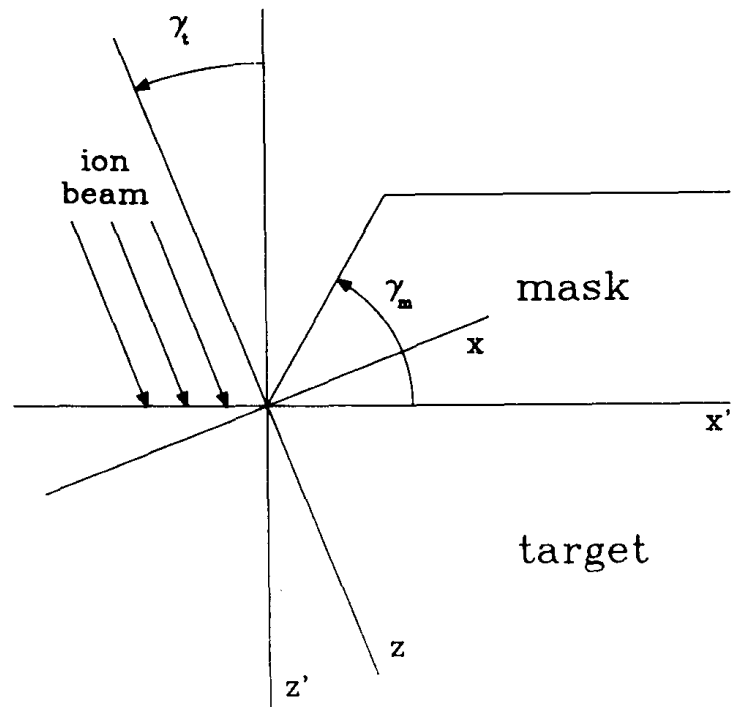

(a)

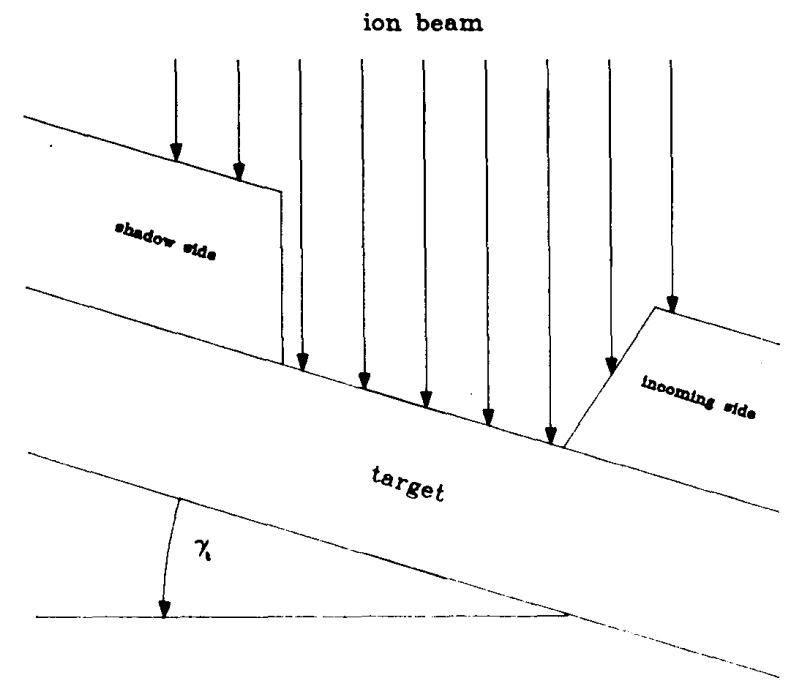

(b)

Fig. 2. (a) Placement of the wafer with the long mask lines in the implanter; (b) a shadow side arises on the left mask edge and an incoming side at the right mask edge depending on the wafer tilt angle and mask angle.

composed of pure hydrofluoric acid with a few drops of 60 -percent nitric acid. Under the influence of light, this etch colors the p-doped areas dark, while the n-doped areas including the depletion layer remain uncolored.

\section{Simulation of Ion Implantation}

Several simulation techniques can be used to calculate the influence of mask edges on the doping profile when using high energy ion implantation. Superposition of a single Point-Response function [14] over the complete simulation area is a rather simple and fast technique which can be easily implemented in a computer program (see 
Fig. 3). This description is very simple and it is possible to derive a mathematical expression for the solution of the convolution integral, therefore this method is very fast. The Point-Response function may be described with a 2-D-Gaussian probability distribution as proposed by $\mathrm{Fu}$ rukawa et al. [15]. A modification to this theory was made by Runge [16] in order to incorporate arbitrary mask shapes. The normalized two-dimensional impurity distribution may be described as [16]

$$
\begin{aligned}
\frac{F(x, z)}{F_{\max }}= & \frac{G(x) G(z)}{F_{\max }}=\frac{1}{\sqrt{2 \pi} \Delta_{x}} \\
& \cdot \int_{-\infty}^{\infty} \exp \left(-\frac{(x-\xi)^{2}}{2 \Delta_{x}^{2}}\right. \\
& \left.-\frac{\left(z-d_{o x}(\xi)-R_{p}\right)^{2}}{2 \Delta_{R_{p}}^{2}}\right) d \xi
\end{aligned}
$$

where $\Delta_{x}$ is the lateral straggle, $\Delta_{R_{p}}$ the projected straggle, and $R_{p}$ the projected range. The shape of the mask is described by $d_{\mathrm{ox}}(x)$. For simplicity, it is assumed here, that the stopping power of the mask material equals the stopping power of silicon. Equation (1) should be modified to incorporate the effect of the tilted wafer [17]. If the mask shape is linear and no ions are assumed to penetrate through the mask outside the edge area, the solution can be given by

$$
\begin{aligned}
\frac{F(x, z)}{F_{\max }}= & \left.\frac{\exp \left(-\frac{\left(x \tan \gamma_{t}-\left(z-R_{p}\right)\right)^{2}}{2 \Delta_{R_{p}}^{2} C_{f}^{2}}\right)}{2 C_{f}}\right) \\
& \cdot\left[1-\operatorname{erf}\left(\frac{x+\tan \gamma_{t}\left(\frac{\Delta_{x}}{\Delta_{R_{p}}}\right)^{2}\left(z-R_{p}\right)}{\sqrt{2} \Delta_{x} C_{f}}\right)\right] \\
& \left.+\frac{\exp \left(-\frac{\left(-x \tan \left(\gamma_{m}-\gamma_{t}\right)-\left(z-R_{p}\right)\right)^{2}}{2 \Delta_{R_{p}}^{2} C_{e}^{2}}\right)}{2 C_{e}}\right) \\
& {\left[\begin{array}{l}
-x+\tan \left(\gamma_{m}-\gamma_{t}\right)\left(\frac{\Delta_{x}}{\Delta_{R_{p}}}\right)^{2}\left(z-R_{p}\right) \\
1-\operatorname{erf})
\end{array}\right] }
\end{aligned}
$$

with

$$
C_{f}=\sqrt{1+\left(\frac{\Delta_{x}}{\Delta_{R_{p}}} \tan \gamma_{t}\right)^{2}}
$$

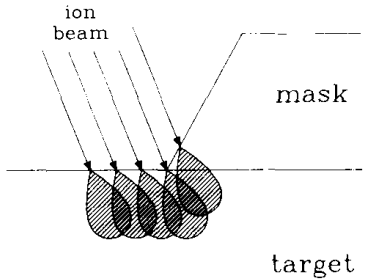

Fig. 3. Simulation of implantation in a target. The doping profile can be calculated by superposition of a single Point-Response function.

and

$$
C_{e}=\sqrt{1+\left(\frac{\Delta_{x}}{\Delta_{R_{p}}} \tan \left(\gamma_{m}-\gamma_{t}\right)\right)^{2}} .
$$

The first team in (2) represents the impurity distribution of a wafer tilted with an angle $\gamma_{t}$. The second term incorporates the effect of the mask edge, with a mask angle $\gamma_{m}$. The above-mentioned equation is embodied in the computer program IMPCAL. Using the transformation,

$$
\begin{aligned}
& x=x^{\prime} \cos \gamma_{t}-z^{\prime} \sin \gamma_{t} \\
& z=z^{\prime} \cos \gamma_{t}-x^{\prime} \sin \gamma_{t}
\end{aligned}
$$

the two-dimensional impurity distribution can easily be computed for arbitrary mask angles and wafer tilt angles. The used values for $R_{p}$ and $\Delta_{R_{p}}$ are 0.995 and $0.195 \mu \mathrm{m}$, respectively [18], and $\Delta_{x}$ equals $\Delta_{R_{p}}$

Although, (2) is a rather rough way to characterize the 2-D doping profile, it will be shown, that the strong asymmetry in high energy ion implantation due to the $7^{\circ}$ wafer tilt is well predicted by it. The Gaussian approximation may not be correct for high energy implantations. A more accurate description might be a Pearson probability distribution in the vertical direction combined with a Gaussian distribution with a depth dependent standard deviation in the lateral direction [19]. To obtain an even better accuracy, Monte Carlo simulations can be used [20], [21]. An advantage of the Monte Carlo method is that ions which leave or scatter from the mask edge can be taken into account. After leaving the mask edge these ions travel through the vacuum and they finally may reenter the target (see Fig. 4). Using a convolution method it is not possible to take these ions into account, it is also not possible to compute the implantation profile if $\gamma_{m}-\gamma_{t}>90^{\circ}$, with $\gamma_{m}$ the angle between the mask edge and the target and $\gamma_{t}$ the tilt angle (see Fig. 2). Especially when $\gamma_{m}-\gamma_{t}=$ $90^{\circ}$ or when light ions are used (like boron), the scattering of ions from the mask edge cannot be neglected anymore. A clear disadvantage of the Monte Carlo method is the required amount of computer time.

The algorithms used for the Monte Carlo simulations are nearly the same as the algorithms used in the TRIM85 program [22]. With our program we can compute the implantation profile near a mask edge. Some simple techniques were used to speed up the Monte Carlo simula- 


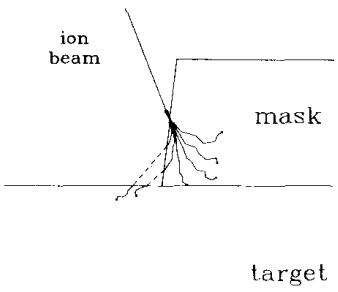

Fig. 4. Possible trajectories of ions implanted at a mask edge. Some ions may leave the mask and travel through vacuum before reentering the target.

tions. A computed table of scatter angles can be used more than once to compute an ion trajectory, rotation symmetry can be used and a computed ion trajectory can be used on several places along the $x$-axis [23]. In our case five trajectories are computed using the same table of scatter angles, each trajectory is rotated $180^{\circ}$ around the implantation axis and each trajectory is used 200 times (number of grid points in $x$-direction) along the $x$-axis. So only one table of scatter angles is used to compute the positions of $5 * 2 * 200=2000$ ions implanted in the simulation area. Ions leaving the mask edge are assumed to move rectilinear through the vacuum without losing energy, these ions may hit the target and then the ion trajectory can be continued in the target (see Fig. 4).

\section{Discussion}

An SEM was used to study the influence of high energy ion implantation on the photoresist mask edges. For this purpose, both implanted and unimplanted wafers are broken in several small samples perpendicular to the series of long photoresist lines. This is done very carefully in order to avoid damaging of the photoresist. The photoresist is brittle and neatly cracks with the silicon wafer. The samples are coated to make them conductive to the electron beam. All samples with implanted and non-implanted photoresist show a nearly perpendicular mask edge; it is expected, that the mask edge angle is about $82-85^{\circ}$. From the SEM photographs we conclude that the shape of the mask edge is not changed due to the high energy implantation.

On the other hand, comparison of the implanted and non-implanted samples shows that the structure of the photoresist is strongly affected by the high energy implantation. It has been reported, that the structure of photoresist changes to disordered graphite by high dose ion implantation [24]. Due to the $1-\mathrm{MeV}$ phosphorus ions, the atomic bonds in the top of the photoresist layer are broken. Here the phosphorus ions lose their energy and come to rest. In the SEM photographs (Fig. 5) a sharp transition in the photoresist can be seen. Below the transition, just a few phosphorus ions come to rest and the photoresist is nearly undamaged. At that place the photoresist breaks as brittle as the sample with non-implanted photoresist. Above the transition the photoresist is fairly damaged by the high energy implanted phosphorus ions.

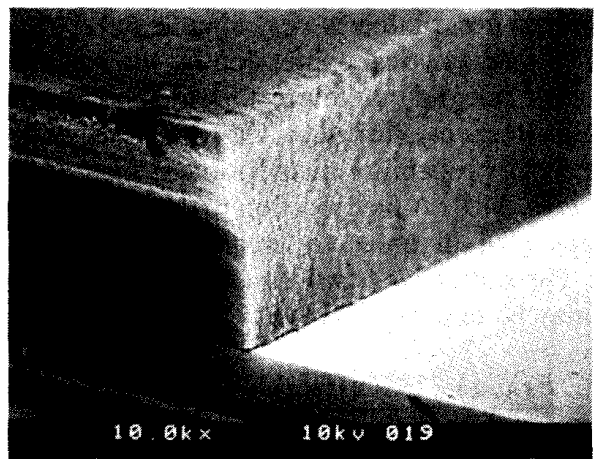

(a)

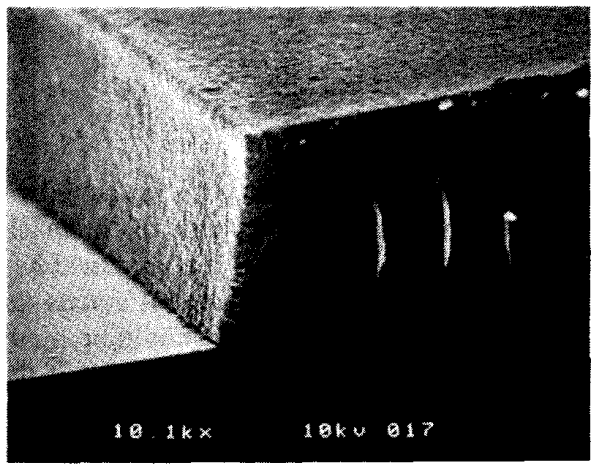

(b)

Fig. 5. SEM photographs of high-energy ion implanted resist after breaking. The transition shows where the photoresist is severely damaged. On the shadow side (a) it is a straight line, whereas on the incoming side, (b) shows a strong curvature.

The shape of the transition in the photoresist depends on the angle between the ion beam and the mask edge and can be compared with the computer plots of the Monte Carlo simulation (see Fig. 6). The results obtained by the SEM and the Monte Carlo simulations seem to be quite similar, the contours in Fig. 6 equal the shape of the transition in the SEM photographs remarkably well. In the case of nearly perpendicular mask edges, the $7^{\circ}$ wafer tilt causes a strong difference between the shadow side and the incoming side. On the incoming side, the transition follows more or less the shape of the mask edge as predicted by the Monte Carlo simulation. On the shadow side the transition between the damaged and undamaged photoresist is almost a straight line as far as to the edge, this is also in agreement with the Monte Carlo simulations.

The ball-grooving technique can be used to measure junction depths. Using this technique the depth information is strongly improved. The result of a ball-grooved buried n-layer is given in Fig. 7. In this figure, the uncolored circle represents the buried n-layer, the colored areas represent the p-doped substrate.

In our case, the ball-grooves are made at the mask edges resulting in an incomplete circle representing the buried $\mathrm{n}$-layer. The part of the circle which is not completed corresponds with the part of the substrate which was covered 


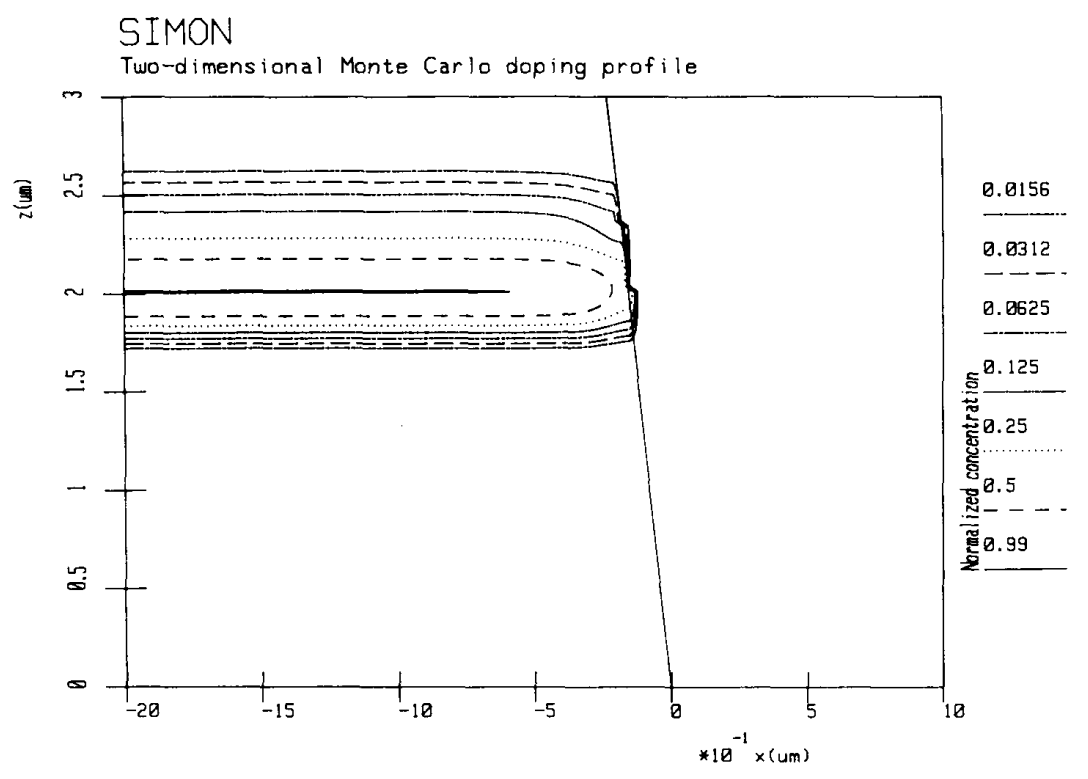

(a)

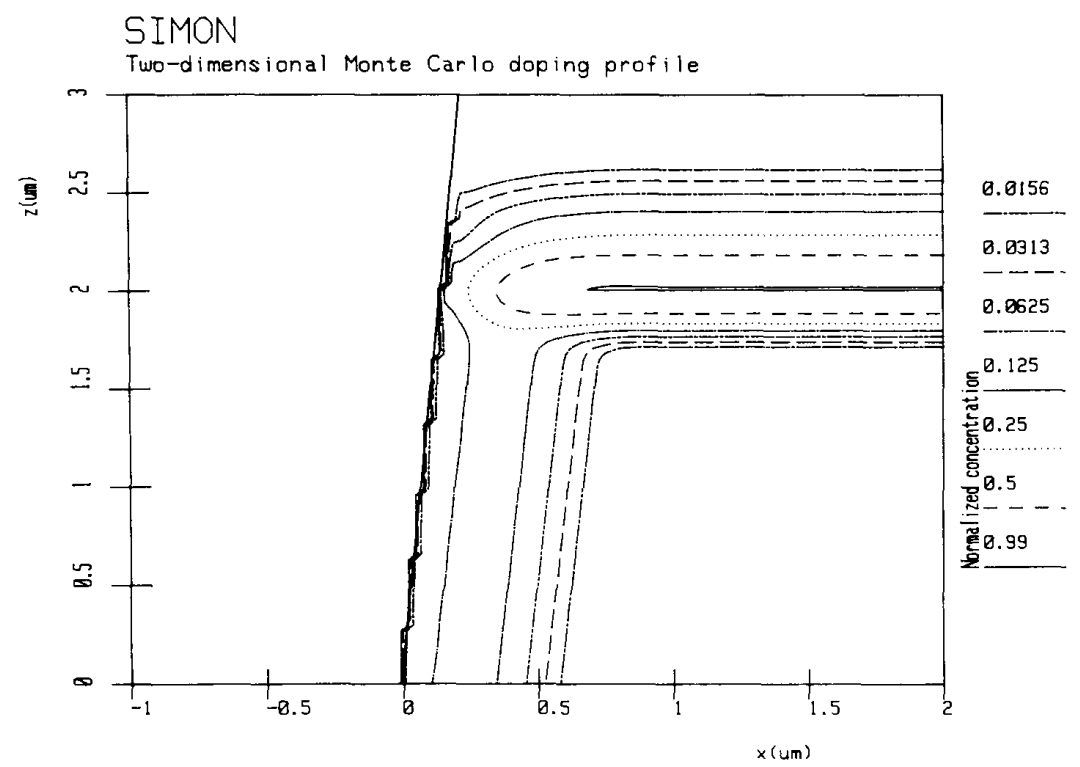

(b)

Fig. 6. Normalized doping profile in the mask of 1-MeV phosphorus implantation on (a) the shadow side and (b) the incoming side as simulated with Monte Carlo. The wafer tilt is $7^{\circ}$ and the mask angle $85^{\circ}$.

with the mask during the ion implantation. In Fig. 8, photographic magnifications of the part of the ball grooves, where the mask edges have been situated, are shown. On the incoming side, the arc, which visualizes the p-doped silicon at the surface, is crossed by an uncolored white trunk at the mask edge. This trunk goes from the buried n-doped layer up to the surface. Thus at this mask edge, the phosphorus ions locally exceed the background boron concentration of $6 \times 10^{16} \mathrm{~cm}^{-3}$. The thickness of this trunk is about $0.8 \mu \mathrm{m}$. On the shadow side, no trunk from the buried n-layer to the surface is observed. Therefore, the $1-\mathrm{MeV}$ phosphorus ions under the mask edge of the shadow side are completely compensated by the back ground doping. This implies, that the $2 \times 10^{13} \mathrm{~cm}^{-2} \mathrm{im}-$ planted phosphorus ions do not result in a highly doped trunk from the buried layer to the surface. In fact the maximum phosphorus concentration in this trunk, if any, remains less than $6 \times 10^{16} \mathrm{~cm}^{-3}$. It is difficult to measure 


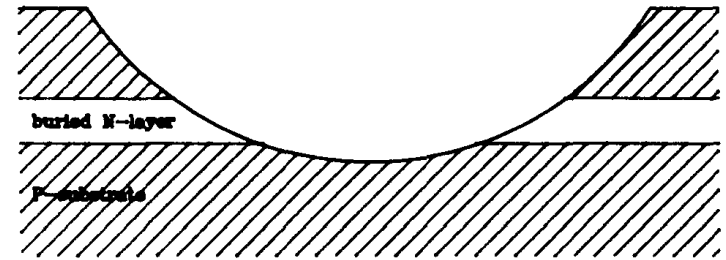

(a)

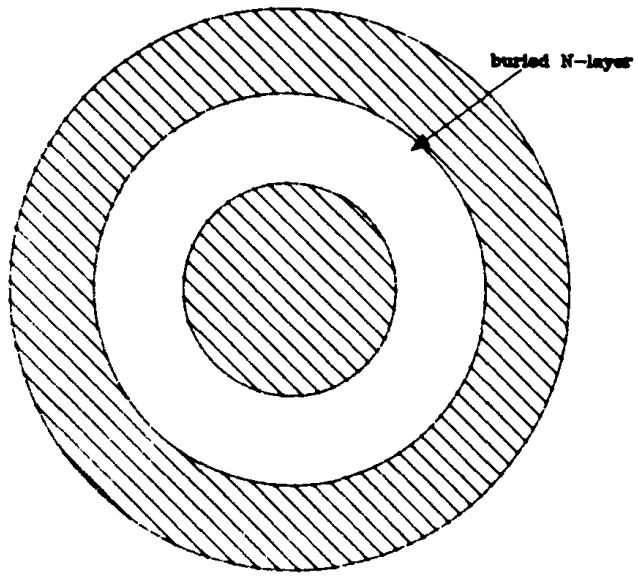

(b)

Fig. 7. Schematic representation of the stained ball-grooves: (a) side view and; (b) top view. The buried n-layer is visible as a white colored circle.

the exact doping profile in the trunk. By tilting the wafer to different angles, lateral information can be gained [25], [26]. However, because of the small thickness of the trunk $(0.8 \mu \mathrm{m})$, medium concentrations and the danger of channeling [8], [9] when implanting into different directions, the concentration in the trunk cannot be measured with SIMS or SAM.

The simulation results very well agree with the experimental results as can be seen in Fig. 9. Both 2-D-Gaussian and Monte Carlo simulations predict a trunk from the buried layer up to the surface on the incoming side. The concentration in this trunk is 20 and 16 percent of the maximum concentration for the Gaussian and Monte Carlo simulation, respectively. On the shadow side, the Monte Carlo shows no trunk, but just a small bulge, as a result of the ions which leave the mask and reenter the target. In spite of the first-order approximation of the theory of Furukawa, the trunk of the Gaussian simulation astonishingly resembles the trunk of the Monte Carlo simulation. Only the small bulge is not predicted by the Gaussian simulation, but the concentration in this bulge is relatively low when compared with the maximum concentration in trunk. On the other side, the Gaussian distribution poorly predicts the lower side of the doping profile in vertical direction. This becomes more pronounced for high energy implantations, in which case a Pearson distribution should give better results [19].

In Fig. 10 the maximum doping concentration in the trunk at the surface normalized to the maximum doping

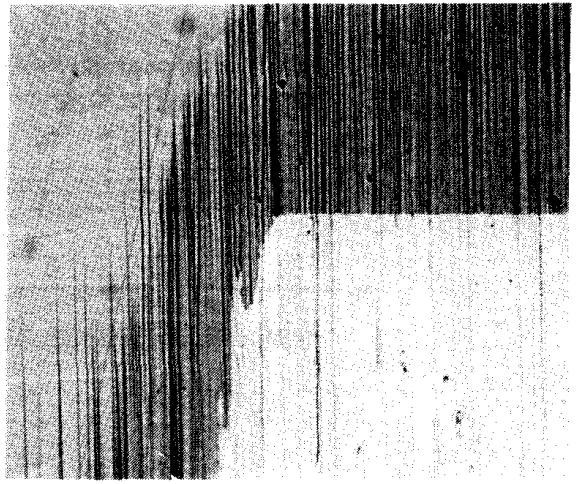

(a)

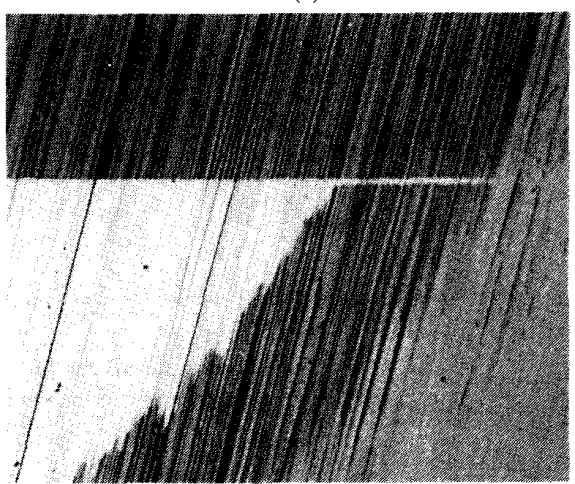

(b)

Fig. 8. Photographic magnifications of the ball-grooves at the mask edges On the shadow side (a) there is no trunk. On the incoming side (b) a trunk is found from the buried n-layer up to the surface.

concentration in the substrate is given as a function of the wafer tilt and for two different mask angles. The relative concentration as simulated with the Gaussian method is about 20-30 percent higher than the concentration, which is simulated with the Monte Carlo method. It can be seen from Fig. 10, that in spite of a smaller wafer tilt, the concentration in the trunk will be considerably high. In the case of an edge of 90 degrees and a wafer tilt of $0^{\circ}$ the Gaussian simulation predicts the absence of a trunk. On the other hand, the Monte Carlo simulation shows that due to the scattering of ions from the mask to the substrate a maximum surface concentration of about 2.2 percent of the maximum concentration occurs. If the wafer tilt is less than $2.5^{\circ}$ then the maximum surface concentration is caused by the scattering of ions. This effect cannot be simulated with the Gaussian method.

\section{Conclusions}

A strongly asymmetric doping profile at the mask edges is found in the case of high energy ion implantation due to the frequently used a $7^{\circ}$ wafer tilt. Neither nearly perpendicular $\left(82-85^{\circ}\right)$ photoresist masks nor fully anisotropical etched oxide masks are able to alleviate this problem.

High energy phosphorus implantations have been done in p-type wafers in order to form buried interconnect. The 


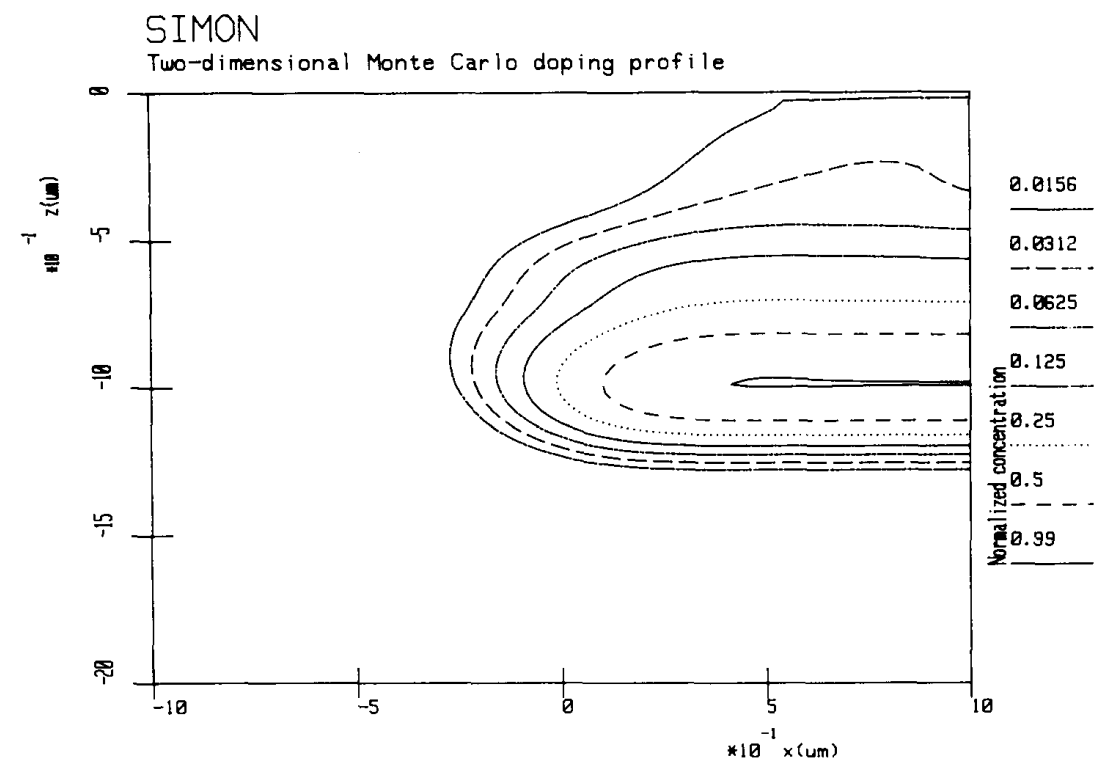

(a)

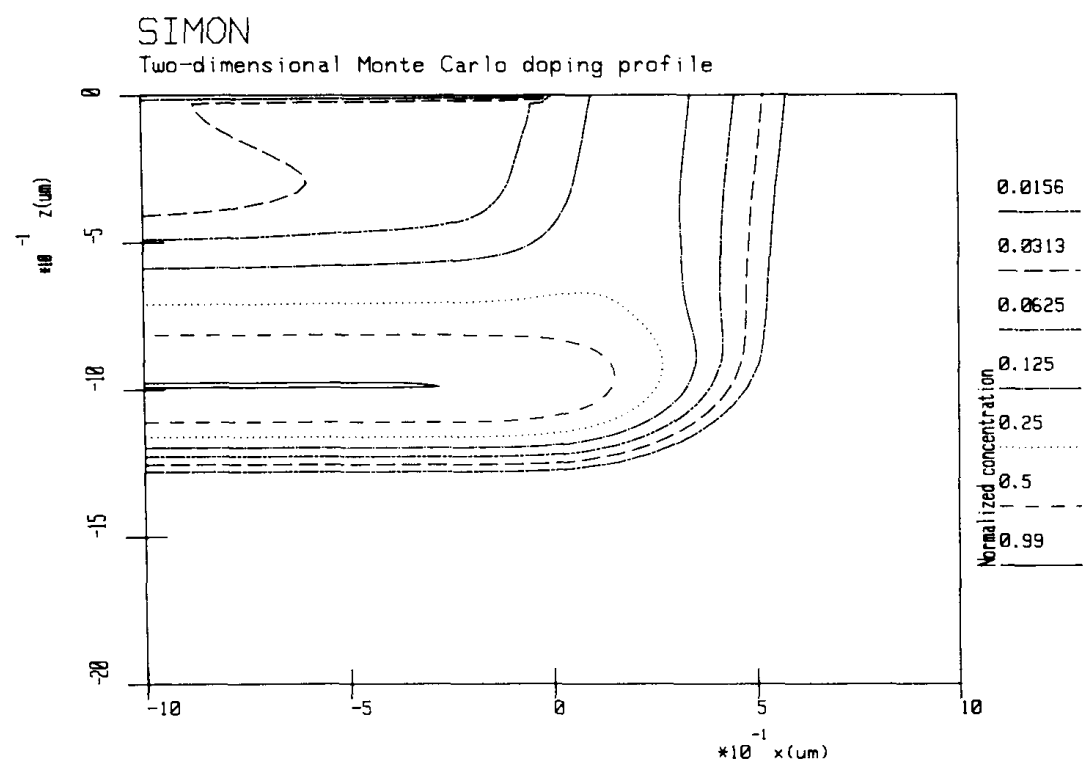

(b)

Fig. 9. Normalized doping profile of 1-MeV phosphorus implantation. On the shadow side (a) a lowly doped bulge is found according to Monte Carlo simulation. On the incoming side, Monte Carlo (b) simulation shows a trunk. (Fig. 9(c) is shown on p. 86).

high energy implanted phosphorus ions create a lot of damage in the photoresist mask, where they come to rest. After breaking the photoresist, this nicely visualizes the asymmetry at the two different mask edges. Besides, the influence of the $7^{\circ}$ wafer tilt on the high energy implanted profile is experimentally shown by means of staining. On the incoming side of the mask edge, a trunk is found from the buried layer to the surface. In the case of a $85^{\circ}$ mask angle, the doping concentration in this trunk is about 20 percent of the maximum concentration in the buried layer. On the shadow side no trunk has been observed. This implies, that the concentration in this trunk, if any, is fully compensated by the background doping.

The existence of this mask edge dependent trunk is also demonstrated by 2-D-Gaussian and Monte Carlo simulations. Both simulations are in excellent agreement with 


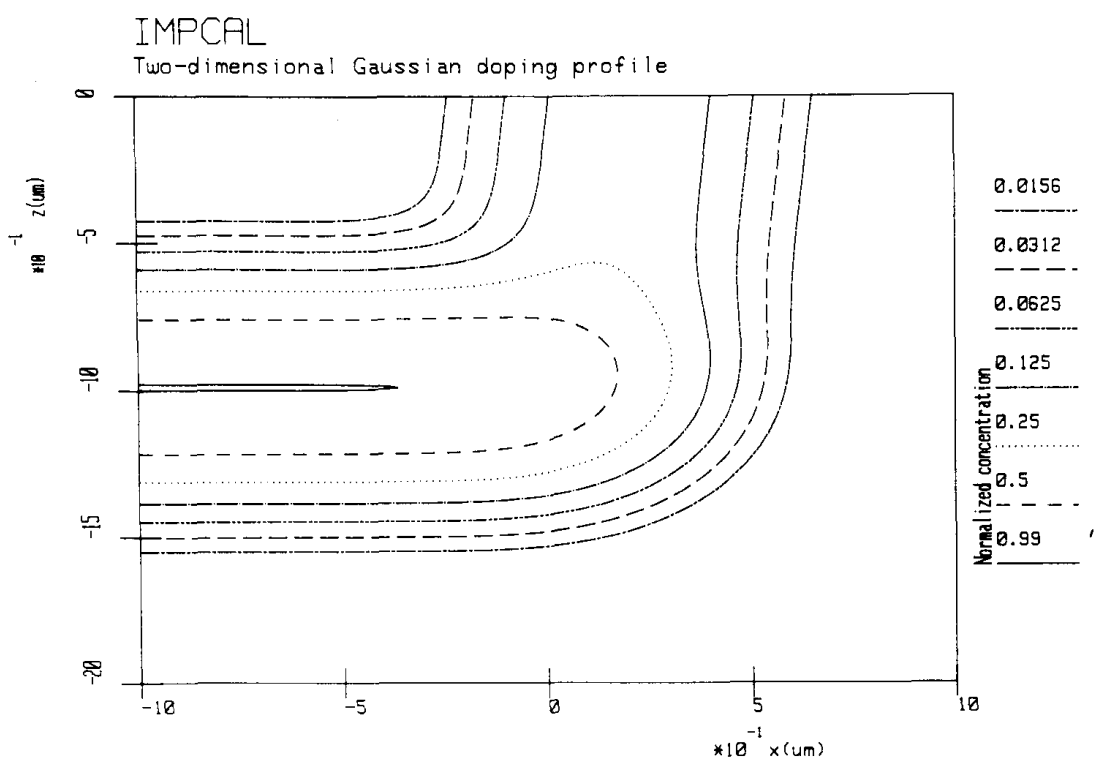

(c)

Fig. 9(c) On the incoming side, Gaussian simulation shows a trunk.

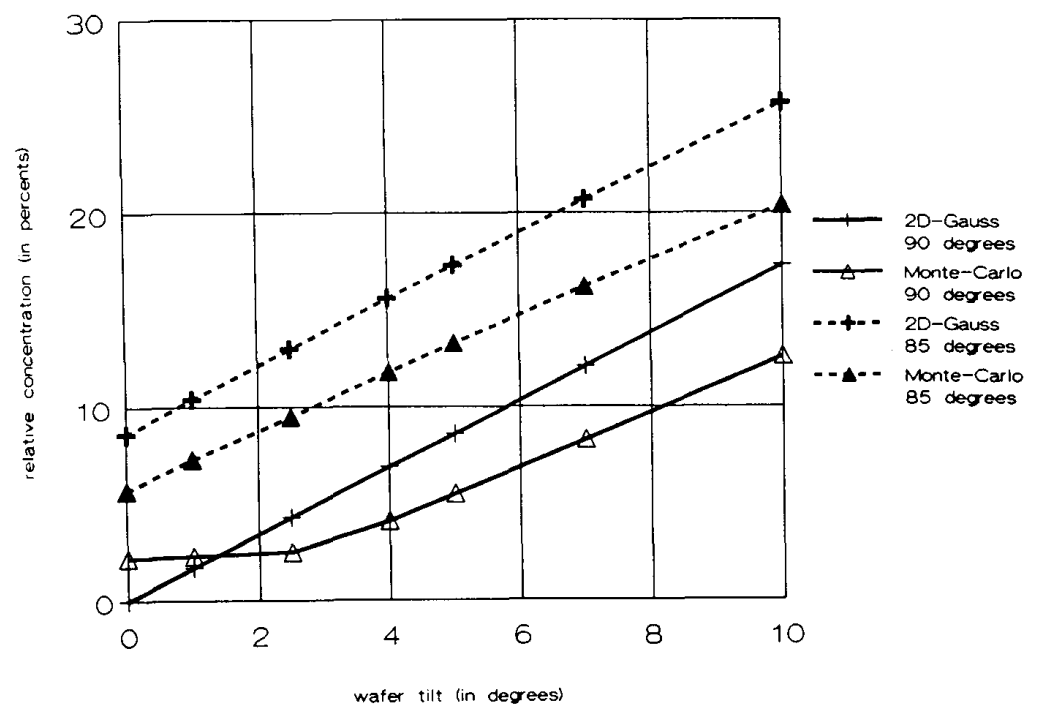

Fig. 10. The normalized maximum doping concentration in the trunk as a function of the wafer tilt for two different angles of the mask edge.

the experimental observed trunks. The trunk as predicted by the Gaussian simulation is in nice agreement with the trunk as predicted by the Monte Carlo simulation.

The observed doping profile asymmetry at the mask edges restricts the application of high energy ion implantations. Buried interconnect in active areas cannot easily be made by simple ion implantation with high energy. In addition, CMOS-processes with retrograde wells may suffer from asymmetric characteristics concerning the holding voltage and the threshold voltage below the field oxide.

\section{ACKNOWLEDGMENT}

The authors wish to thank E. van Schie and T. Aarnink for their helpful suggestions.

\section{REFERENCES}

[1] R. D. Rung, C. J. Dell'Oca, and L. G. Walker, "A retrograde p-well for higher density CMOS," IEEE Trans. Electron Devices, vol. ED-28, pp. 1115-1119, 1981.

[2] J. Y. Chen, "Quadruple-well CMOS for VLSI technology," IEEE Trans. Electron Devices, vol. ED-31, pp. 910-919, 1984.

[3] Y. Taur, et al., "A self-aligned 1- $\mu$ m-channel CMOS technology with 
retrograde n-well and thin epitaxy," IEEE Trans. Electron Devices, vol. ED-32, pp. 203-209, 1985.

[4] A. Stolmeijer, "A twin-well CMOS process using high energy ion implantation,' IEEE Trans. Electron Devices, vcl. ED-33, pp. 450$457,1986$.

[5] R. Koch, H. Herbst, and P. Jespers, "Three-terminal CID as random access memory cell," IEEE J. Solid State Circuits, vol. SC-12, pp. $534-536,1977$.

[6] J. Lohstroh, J. J. M. Koomen, A. T. van Zanten, and R. H. W. Salters, "Punch-through currents in $\mathrm{P}^{+} \mathrm{NP}^{+}$and $\mathrm{N}^{+} \mathrm{PN}^{+}$sandwich structures-I," Solid State Electron., vol. 24, no. 9, pp. 805-814, 1981 .

[7] T. Mouthaan and M. Vertregt, "A vertically integrated dynamic RAM-cell: buried bit line memory cell with floating transfer layer," Solid State Electron., vol. 29, no. 12, pp. 1289-1294, 1986.

[8] M. I. Current, N. L. Turner, T. C. Smith, and D. Crane, "Planar channeling effects in Si(100)," Nucl. Instrum. Methods, vol. B6, pp. $336-348,1985$

[9] K. Cho, et al. "Channeling effect for low energy ion implantation in Si,' Nucl. Instrum. Methods, vol. B7, pp. 265-272, 1985.

[10] P. Spinelli, A. M. Carter, and M. Bruel, "Critical aspects of high energy implants for CMOS technology: Channeling effects and masking problems,' Nucl. Instrum. Methods, vol. B21, pp. 452-455, 1987.

[11] Ion Implantation. New York: Academic, J. F. Ziegler, Ed., 1988.

[12] P. Spinelli, J. Escaron, A. Soubie, and M. Bruel, "High energy ion implantation for C-MOS isolation $\mathbf{n}$-wells technology: problems related to the use of multicharged phosphorus ions in an industrial context," Nucl. Instrum. Methods, vol. B6, pp. 283-286, 1985.

[13] M. G. Pitt and P. A. van der Plas, "Limitations on $n+/ p+$ spacing due to shadowing effects in a $0.7 \mu \mathrm{m}$ retrograde well cmos process," in 18th ESSDERC, Sept. 1988, pp. 553-556.

[14] M. D. Giles, "Calculation of ion implantation profiles for two-dimensional process modeling," in Proc. 2nd Conf. Simulation Semiconductor Devices Processes, 1986, pp. 233-246.

[15] S. Furukawa, H. Matsumura, and H. Ishiwara, "Theoretical considerations on lateral spread of implanted ions," Japan. J. Appl. Phys., vol. 11 , no. 2 , pp. 134-142, 1972.

[16] H. Runge, "Distribution of implanted ions under arbitrarily shaped mask edges," Phys. Stat. Sol. A, vol. 39, pp. 595-599, 1977.

[17] T. Tsuchiya, "Tilt angle and mask edge angle dependences of lateral spread of implanted boron ions under mask edge," J. Appl. Phys., vol. 51 , no. 11 , pp. 5773-5780, 1980.

[18] S. Oosterhoff, "Distribution of boron and phosphorus implanted in silicon in the energy range 0.1-1.5MeV," Nucl. Instrum. Methods, vol. B30, pp. 1-12, 1988.

[19] G. Hobler, E. Langer, and S. Selberherr, "Two-dimensional modeling of ion implantation," in Proc. 2nd Conf. Simulation Semiconductor Devices Processes, 1986, pp. 256-270.

[20] T. Ishitani, K. Murata, and R. Shimizu, "Monte Carlo simulations of the behaviour of energetic light ions in solids," Japan. $J$. Appl. Phys., vol. 10. no. 10, p. 1464, 1971.

[21] T. Ishitani, R. Shimizu, and K. Murata, "Monte Carlo simulations on the behaviour of energetic ions in polyatomic targets," Phys. Stat. Sol. B, vol. 50, pp. 681-690, 1972

[22] J. F. Ziegler, J. P. Biersack, and U. Littmark, "The Stopping and Range of lons in Solids. New York: Pergamon, 1985.

[23] E. van Schie and J. Middelhoek, "Two methods to improve the performance of Monte Carlo simulations of ion implantations in amorphous targets," IEEE Trans. Computer-Aided Design, vol. 8, pp. 108-113, Feb. 1989
[24] Y. Okuyama, T. Hashimoto, and T. Koguchi, "High dose ion implantation into photoresist," J. Electrochem. Soc., vol. 125, no. 8 , pp. 1293-1298, 1978

[25] S. Furukawa and H. Matsumura, "Backscattering study on lateral spread of implanted ions," Appl. Phys. Lett., vol. 22, no. 3, pp. 97 98,1973

[26] E. van Schie, J. Middelhoek, and P. C. Zalm, "Determination of 2D implanted ion distributions using inverse radon transform methods,' IEEE Trans. Computer-Aided Design, to be published.

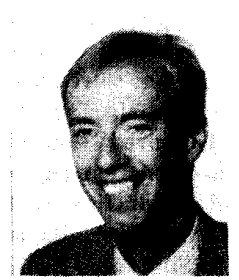

Rutger C. Wijhurg was born in Nijmegen, The Netherlands in 1962. He received the M.Sc. de gree in electrical engineering from the University of Twente, Enschede, The Netherlands, in 1986. Currently, he is working toward the Ph.D. degree at the University of Twente.

His research has been concentrated on the design of SRAM's. In 1985, he joined Siemens, Munich, where the was engaged in the development of chip cards. His research topics include combined bipolar-CMOS processing using high energy ion implantation, the application of BiCMOS circuits, new device structures, and device modeling.

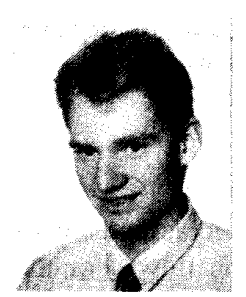

Gertjan J. Hemink was born in Wisch, The Netherlands, on December 7,1964 . He received the M.Sc. degree in electrical engineering from the University of Twente, Enschede, The Netherlands, in 1988. Since March 1988, he has been working toward the Ph.D. degree at the University of Twente.

At present his main research interests are the development and application of new EEPROM structures using high energy ion implantation. Hi general interests are in the field of device deve opment and application, advanced 2-D process and device simulation tools and new technologies and materials.

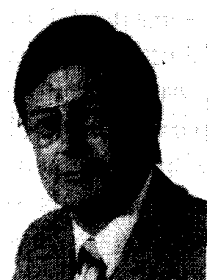

Jan Middelhoek was born in Rotterdam in 1935 He received the M.Sc. degree from the Free University, Amsterdam, The Netherlands, and the D.Sc. degree from the State University, Leiden. $\mathrm{He}$ is a professor at the University of Twente, The Netherlands, where he teaches IC technology and electronics. He is the head of the Laboratory for Integrated Circuits. His research is concentrated on the applications of $\mathrm{MeV}$ ion implantation in IC fabrication. He is a member of the German/Dutch MEGA expert committee for the development of submicron-technology and chairman of the Semiconductors' group of the Foundation of Fundamental Research on Matter (FOM) Utrecht. He is also a coordinator of a student mobility program in the field of microelectronics in the framework of Euramusprogram of the E.E.C. 